\title{
A Logic for Global and Local Announcements
}

\author{
Francesco Belardinelli \\ Labortoire IBISC, UEVE \\ and IRIT Toulouse \\ belardinelli@ibsc.fr
}

\author{
Hans van Ditmarsch \\ LORIA - CNRS \\ Université de Lorraine \\ Vandoeuvre-lès-Nancy, France \\ hvd@us.es
}

\author{
Wiebe van der Hoek \\ Department of Computing \\ University of Liverpool \\ Liverpool, UK \\ wiebe@csc.liv.ac.uk
}

\begin{abstract}
In this paper we introduce global and local announcement logic (GLAL), a dynamic epistemic logic with two distinct announcement operators - $[\phi]_{A}^{+}$and $[\phi]_{A}^{-}$indexed to a subset $A$ of the set $A g$ of all agents - for global and local announcements respectively. The boundary case $[\phi]_{A g}^{+}$corresponds to the public announcement of $\phi$, as known from the literature. Unlike standard public announcements, which are model transformers, the global and local announcements are pointed model transformers. In particular, the update induced by the announcement may be different in different states of the model. Therefore, the resulting computations are trees of models, rather than the typical sequences. A consequence of our semantics is that modally bisimilar states may be distinguished in our logic. Then, we provide a stronger notion of bisimilarity and we show that it preserves modal equivalence in GLAL. Additionally, we show that GLAL is strictly more expressive than public announcement logic with common knowledge. We prove a wide range of validities for GLAL involving the interaction between dynamics and knowledge, and show that the satisfiability problem for GLAL is decidable. We illustrate the formal machinery by means of detailed epistemic scenarios.
\end{abstract}

\section{Introduction}

In this paper we take inspiration from the state of the art in public announcement logic (PAL) and introduce a logic for global as well as local announcements. Public announcement logic has two key features. First, announcements are public, in the sense that all agents equally observe the new information, and are (commonly) aware of all equally observing the information. Second, announcements are global, that is, although for truthful public announcements the truth of the announced formula in the actual state is a precondition, how the new information is processed does not depend on the actual state but rather on the model (i.e., public announcements are model transformers).

In the proposed framework we carefully distinguish the two, independent features of publicity and globality, which are packed together in the announcement operator $[\phi]$, and relax both of them. Hence, by weakening publicity, we allow to make announcements to a proper subset $A$ of the set $A g$ of all agents. Then, only the agents in $A$ partake of the new information contained in the announcement. Further, by weakening globality, we distinguish between local announcements, whose meaning depends on the actual state, and global announcements that depend on general features of the model.

As a result, the language of global and local announcement logic (GLAL) contains two modalities $[\phi]_{A}^{+}$and $[\phi]_{A}^{-}$, for the global and local announcement of formula $\phi$ respectively, each of them indexed to a coalition $A$ of agents. We endow GLAL with a semantics in terms of pointed model updates that reflects the intuitions illustrated above. Most interestingly, we are able to provide a unified account of both global and local announcements, in which the difference between the two depends on a subtle distinction in the update mechanism.

Related Work. Public announcement logics have witnessed a wealth of contributions in recent years [21, 11, 6, 10], thus making virtually impossible to give an exhaustive account of this research area. Here

J. Lang (Ed.): TARK 2017

EPTCS 251, 2017, pp. 28-42 doi 10.4204/EPTCS.251.3 (c) F. Belardinelli, H. van Ditmarsch \& W. van der Hoek

This work is licensed under the Creative Commons Attribution License. 
we mention the references most closely related to the present endeavour, as well as some surveys on PAL. In [16] a logic of fully private announcements was proposed, while [9, 18, 3, 19] put forward logics of semi-private announcements, which relax the publicity assumption of PAL in various directions. Such private or semi-private announcements have also been modelled as action models [4]. Differently from our proposal, in semi-private announcements the agents that do not observe the announcement of $\phi$ learn at least that the other agents have learnt whether $\phi$. No such assumption holds in the present context. On the other hand, in fully private announcements the other agents learn nothing at all about the agents learning $\phi$ (which is typically interpreted as the other agents not even being aware of the announcement having taken place). This is also different from our setting, wherein these other agents learn something about $\phi$ w.r.t. the actual state.

Modal logics based on model transformations have also been proposed in [2, 5, 15, 1]. These accounts share the aspect of locality (dependence of the model transforming operation on the actual state) that also characterizes our approach. However, differently from our proposal, these are very expressive formalisms (typically undecidable, or non-axiomatizable) that allow to add or remove individual pairs of states from an agent's accessibility relation; thus operating on a purely semantic level. On the contrary, in GLAL the model transformation is determined by the announced formula, so that only pairs satisfying a condition relative to this formula can be removed. Our logic is therefore decidable, although we have not yet provided an axiomatization. We also provide results on bisimulations and the model checking problem.

Schema of the paper. In Section 2 we introduce the syntax and semantics of GLAL and provide two examples to illustrate the formalism. In Section 3 we compare our approach to relevant related account appearing in the literature on dynamic epistemic logic (DEL); while in Sections 4 and 5 we analyse the expressivity of GLAL and prove that, differently from PAL, GLAL cannot be reduced to epistemic logic (with common knowledge) as it is strictly more expressive. In Section 6 we introduce bisimulation relations that preserve GLAL formulas; whereas in Section 7 we present results on the model checking and satisfiability problems. We discuss the meaning and relevance of these results in Section 8, where we also point to directions for future research.

\section{A Unified Framework for Global and Local Announcements}

In this section we introduce the syntax and semantics of global and local announcement logic. We warn that the term 'announcement' is used here with a different meaning with respect to public announcement logic. As discussed in the introduction, the announcements of PAL appear here as global announcements to all agents. Hence, our notion of announcement is more general as it also covers local announcements and announcement to only a selected subset of all agents. The distinction will be clear once the appropriate semantics is introduced.

Syntax. In the rest of the paper we assume a set $A P$ of atomic propositions (or atoms), and a finite set $A g$ of indexes for agents.

Definition 1 (GLAL) The formulas in GLAL are defined in BNF as follows, for $p \in A P$ and $A \subseteq A g$ :

$$
\psi \quad::=p|\neg \psi| \psi \wedge \psi\left|C_{A} \psi\right|[\psi]_{A}^{+} \psi \mid[\psi]_{A}^{-} \psi
$$

The language $\mathscr{L}_{\text {glal }}$ of GLAL contains epistemic formulas $C_{A} \phi$, for coalition $A \subseteq A g$ of agents, which intuitively says that " $\phi$ is common knowledge in coalition $A$ ", as customary. Moreover, we have global announcement formulas $[\psi]_{A}^{+} \phi$, whose reading is that "after globally announcing $\psi$ to the agents in $A, \phi$ 
is true", as well as local announcements $[\psi]_{A}^{-} \phi$, whose meaning is that "after locally announcing $\psi$ to the agents in $A, \phi$ is true". We will illustrate and discuss, using our semantics, the different interpretations of operators $[\psi]_{A}^{+}$and $[\psi]_{A}^{-}$. The individual knowledge formulas $K_{a} \phi$ can be defined as $C_{\{a\}} \phi$ as standard, as well as symbols $\perp, \top$, connectives $\vee, \rightarrow$, and dual operators $M_{a},\langle\psi\rangle_{A}^{-}$, and $\langle\psi\rangle_{A}^{+}$. Also, the "everybody knows" formula $E_{A} \phi$ is a shorthand for $\bigwedge_{a \in A} K_{a} \phi$. We omit subscript $A$ from $E_{A} \phi$ and $C_{A} \phi$ whenever $A$ is the grand coalition $A g$, and simply write $E \phi, C \phi$. Finally, we write $K w_{a} \phi$ as a shorthand for $K_{a} \phi \vee K_{a} \neg \phi$.

Global and local announcement logic extends a number of well-known formalisms. The language $\mathscr{L}_{\mathrm{pal}^{+}}$obtained by Def. 1 without clause $[\psi]_{A}^{-} \psi$ is (an extension of) public announcement logic; the language $\mathscr{L}_{e l}$ without clause $[\psi]_{A}^{+} \psi$ as well is epistemic logic (with common knowledge), and language $\mathscr{L}_{p l}$ without clause $C_{A} \psi$ as well is propositional logic. These (syntactic) language inclusions can be summarised as follows:

$$
\mathscr{L}_{p l} \subseteq \mathscr{L}_{e l} \subseteq \mathscr{L}_{\text {pal }}{ }^{+} \subseteq \mathscr{L}_{\text {glal }}
$$

Hereafter, when we refer to "epistemic logic", we mean language $\mathscr{L}_{e l}$ including common knowledge.

Semantics. We interpret formulas in GLAL on multi-modal Kripke frames and models, and then define appropriate update mechanisms for global and local announcements.

Definition 2 (Frame) A Kripke frame is a tuple $\mathscr{F}=\left\langle W,\left\{R_{a}\right\}_{a \in A g}\right\rangle$ where

- $W$ is a set of possible worlds;

- for every agent index $a \in A g, R_{a} \subseteq 2^{W \times W}$ is an equivalence relation on $W$.

As customary in epistemic logic [20, 7], for every agent $a \in A g, R_{a}$ is the corresponding indistinguishability relation between worlds in $W$. In the following, for a coalition $A \subseteq A g$, we consider also the reflexive and transitive closure $R_{A}^{C}=\left(\bigcup_{a \in A} R_{a}\right)^{*}$ of the union of indistinguishability relations, for the interpretation of common knowledge. Then, for coalition $A$ and $w \in W$, we set $R_{A}^{C}(w)=\left\{w^{\prime} \mid R_{A}^{C}\left(w, w^{\prime}\right)\right\}$. Notice that, each $R_{a}$ being an equivalence relation, each $R_{A}^{C}(w)$ is the equivalence class of $w \in W$. Moreover, whenever $A$ is a singleton $\{a\}$, we obtain that $R_{A}^{C}(w)=\left\{w^{\prime} \mid R_{\{a\}}\left(w, w^{\prime}\right)\right\}=R_{a}(w)$, and $R_{A}^{C}$ can be represented as the set $\mathscr{E}_{A}=\left\{R_{A}^{C}(w) \mid w \in W\right\}$ of its equivalence classes.

To assign a meaning to formulas in GLAL we introduce assignments as functions $V: A P \rightarrow 2^{W}$. A (Kripke) model is then defined as a pair $\mathscr{M}=\langle\mathscr{F}, V\rangle$.

Definition 3 (Satisfaction) We inductively define the satisfaction set $[\varphi]] \mathscr{M} \subseteq W$ of formula $\varphi$ in model $\mathscr{M}=\langle\mathscr{F}, V\rangle$ as follows:

$\llbracket[p]_{\mathscr{M}} \quad=\quad V(p)$

$\left.\llbracket \neg \psi]]_{\mathscr{M}}=W \backslash[\psi]\right]_{\mathscr{M}}$

$\left.\left.\llbracket \psi \wedge \psi^{\prime}\right]_{\mathscr{M}}=\llbracket[\psi]_{\mathscr{M}} \cap \llbracket \psi^{\prime}\right]_{\mathscr{M}}$

$\left.\llbracket\left[C_{A} \psi\right]_{\mathscr{M}}=\left\{w \in W \mid \text { for all } w^{\prime} \in R_{A}^{C}(w), w^{\prime} \in \llbracket \psi \psi\right]_{\mathscr{M}}\right\}$

$\left.\left.\llbracket[\psi]_{A}^{-} \psi^{\prime}\right]\right]_{\mathscr{F}} \quad\{w \in W \mid \text { if } w \in[\mid \psi]]_{\mathscr{M}}$ then $\left.w \in\left[\left[\psi^{\prime}\right]\right]_{\mathscr{M}_{(w, \psi, A)}^{-}}\right\}$

$\left.\left.\llbracket[\psi]_{A}^{+} \psi^{\prime}\right]\right]_{\mathscr{C}} \quad\left\{w \in W \mid\right.$ if $w \in[[\psi]]_{\mathscr{M}}$ then $\left.w \in\left[\left[\psi^{\prime}\right]\right]_{\mathscr{M}_{(w, \psi, A)}^{+}}^{+}\right\}$

where refinements $\mathscr{M}_{(w, \psi, A)}^{-}=\left\langle W^{-},\left\{R_{a}^{-}\right\}_{a \in A g}, V^{-}\right\rangle$and $\mathscr{M}_{(w, \psi, A)}^{+}=\left\langle W^{+},\left\{R_{a}^{+}\right\}_{a \in A g}, V^{+}\right\rangle$of model $\mathscr{M}$ w.r.t. world $w$, formula $\psi$, and coalition $A$, are defined as

- $W^{-}=W^{+}=W$ and $V^{-}=V^{+}=V$;

- for every agent $b \notin A, R_{b}^{-}=R_{b}^{+}=R_{b}$; while for $a \in A$,

$$
R_{a}^{-}(v)= \begin{cases}R_{a}(v) \cap[[\psi]]_{\mathscr{M}} & \text { if } v \in R_{a}(w) \cap[[\psi]]_{\mathscr{M}} \\ \left.R_{a}(v) \cap[\neg \psi]\right]_{\mathscr{M}} & \text { if } \left.v \in R_{a}(w) \cap[\neg \psi]\right]_{\mathscr{M}} \\ R_{a}(v) & \text { otherwise }\end{cases}
$$




$$
R_{a}^{+}(v)= \begin{cases}R_{a}(v) \cap[[\psi]]_{\mathscr{M}} & \text { if } v \in R_{A}^{C}(w) \cap[[\psi]]_{\mathscr{M}} \\ \left.R_{a}(v) \cap[\neg \psi]\right]_{\mathscr{M}} & \text { if } v \in R_{A}^{C}(w) \cap[[\neg \psi]]_{\mathscr{M}} \\ R_{a}(v) & \text { otherwise }\end{cases}
$$

By Def. 3 the refinement $\mathscr{M}_{(w, \psi, A)}^{-}$only affects worlds that are accessible by each agent in $A$ separately, while $\mathscr{M}_{(w, \psi, A)}^{+}$involves all worlds reachable through relation $R_{A}^{C}$. In all these worlds the accessibility relation is updated according to whether the world in question satisfies the announcement, that is, the announcement refines the equivalence class of each such world. In Example 1 and 2 below we illustrate the differences between the two types of refinement. Notice that in the case of single agents, the refinements $\mathscr{M}_{(w, \psi, a)}^{-}$and $\mathscr{M}_{(w, \psi, a)}^{+}$coincide, hence we omit superscripts - and + from single-agent refinements and modalities. Indeed, globally or locally announcing a fact to a single agent is tantamount, as she is the only one to witness the announcement. In such a case, model refinement $\mathscr{M}_{(w, \psi, a)}$ can be interpreted as "in $R_{a}(w)$ agent $a$ learns whether $\psi$ ". As a consequence, formula $[\psi]_{a} \phi$ then becomes: if $\psi$ holds and $a$ learns whether $\psi$, then $\phi$ holds as well. Also, the updated set $\mathscr{E}_{a}^{\prime}$ of equivalence classes in $\mathscr{M}_{(w, \psi, a)}$ can be shown to be equal to $\left.\left.\left.\left(\mathscr{E}_{a} \backslash\left\{R_{a}(w)\right\}\right) \cup\left\{R_{a}(w) \cap \llbracket \psi\right]\right], R_{a}(w) \cap[\neg \psi]\right]\right\}$.

We introduce standard notions of truth and validity. A formula $\phi$ is satisfied at $w$, or $(\mathscr{M}, w) \models \phi$, iff $w \in[[\phi]]_{\mathscr{M}} ; \phi$ is true at $w$, or $(\mathscr{F}, w) \mid=\phi$, iff $(\langle\mathscr{F}, V\rangle, w)=\phi$ for every assignment $V ; \phi$ is valid in a frame $\mathscr{F}$, or $\mathscr{F} \models \phi$, iff $(\mathscr{F}, w) \models \phi$ for every world $w$ in $\mathscr{F}$. We often omit the subscript $\mathscr{M}$ from the satisfaction set $[[\psi]]_{\mathscr{M}}$ whenever clear by the context.

We now state that our model refinements are well-defined as both $R_{a}^{-}$and $R_{a}^{+}$are actually equivalence relations.

Proposition 1 Let $\mathscr{M}$ be a model with refinements $\mathscr{M}_{(w, \psi, A)}^{-}$and $\mathscr{M}_{(w, \psi, A)}^{+}$. For every agent a $\in A g$, if $R_{a}$ is an equivalence relation, then also $R_{a}^{-}$and $R_{a}^{+}$are.

We refer to Section 3 for a thorough comparison with related approaches to public and private announcements in DEL. Here, we illustrate the difference between global and local announcements by means of two examples drawn from the literature on multi-agent systems and dynamic logic [13, 14]. Hereafter we often represent a set as some sequence of its elements.

Example 1 Here we consider the well-known puzzle of the muddy children. We assume familiarity with this scenario and refer to [12 14] for a detailed presentation. The initial model $\mathscr{M}$ for 3 children (red, blue, and green), where no child knows whether she is muddy, can be represented as follows:

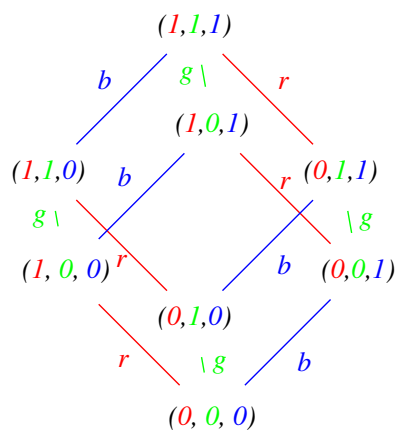

Now suppose that only red is muddy, i.e., the actual world is $(1,0,0)$, and the father locally announces to red, green, and blue that at least one child is muddy, that is, he announces that formula $\alpha:=m_{r} \vee$ $m_{b} \vee m_{g}$ is true. The updated model $\mathscr{M}_{(100, \alpha, r g b)}^{-}$is then given as follows, on the left: 


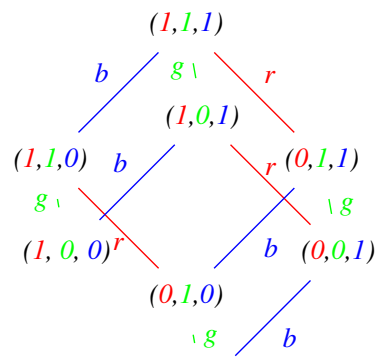

$(0,0,0)$

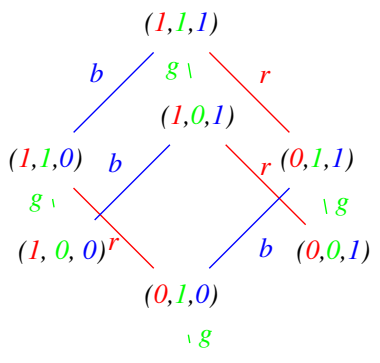

$(0,0,0)$

Notice that only the indistinguishability relation for red is updated, as in all worlds that blue and green consider possible from $(1,0,0)$, formula $\alpha$ is indeed true. Hence, after the father's local announcement, in $(1,0,0)$ all children know that at least one child is muddy, i.e., $(\mathscr{M},(1,0,0)) \models[\alpha]_{r g b}^{-} E \alpha$. Moreover, red learns that she is muddy, i.e., $(\mathscr{M},(1,0,0)) \models[\alpha]_{\text {rgb }}^{-} K_{r} m_{r}$.

On the other hand, the father's local announcement is not enough to make $\alpha$ common knowledge for red, green and blue, that is, $(\mathscr{M},(1,0,0)) \not \models[\alpha]_{r g b}^{-} C_{r g b} \alpha$ as, for instance, blue considers possible that red considers possible that blue considers possible that no child is muddy, that is, $(\mathscr{M},(1,0,0) \models$ $[\alpha]_{r g b}^{-} M_{b} M_{r} M_{b} \neg \alpha$ via epistemic path $(1,0,0) \sim_{b}(1,1,0) \sim_{r}(0,1,0) \sim_{b}(0,0,0)$. This is in contrast with the classic version of the muddy children puzzle with public announcements. In general, for every state $s \in\{0,1\}^{3}$ different from $(0,0,0)$, announcing privately $\alpha$ in s is not sufficient to derive common knowledge of $\alpha:(\mathscr{M}, s) \not \models[\alpha]_{r g b}^{-} C_{r g b} \alpha$

Now suppose that at the beginning, again in world $(1,0,0)$, the father globally announces to red and blue only that at least one child is muddy. The updated model $\mathscr{M}_{(100, \alpha, r b)}^{+}$is shown above on the right. Specifically, in $\mathscr{M}_{(100, \alpha, r b)}^{+}$the indistinguishability relations for both red and blue are updated, and as a result, after the father's global announcement, in $(1,0,0)$ red and blue have common knowledge that at least one child is muddy: $(\mathscr{M},(1,0,0)) \models[\alpha]_{r b}^{+} C_{r b} \alpha$. However, also in this case the father's global announcement is not enough to make $\alpha$ common knowledge amongst all children, that is, $(\mathscr{M},(1,0,0)) \not \forall[\alpha]_{r b}^{+} C_{r g b} \alpha$.

Example 2 Here we consider a simple scenario of communication between a sender $s$ and a receiver $r$ over a reliable channel that is listened to by eavesdropper $e$. The initial state is represented by the following model $\mathscr{N}$, in which s knows the actual value of the bit (either 0 or 1), while $r$ and e are unsure about it.

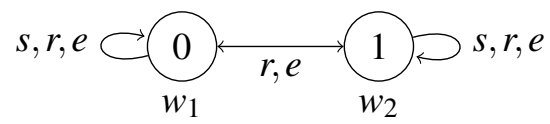

Then, after s has communicated to $r$ the value of the bit, we obtain the updated model $\mathscr{N}_{\left(w_{1}, b i t=0, r\right)}$ :

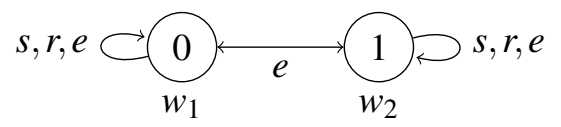

Hence, we have that $\left(\mathscr{N}, w_{1}\right) \models[\text { bit }=0]_{r} K_{r}($ bit $=0)$, as expected. On the other hand, the eavesdropper does not learn the value of the bit, but she learns that $r$ knows it: $\left(\mathscr{N}, w_{1}\right) \models[\text { bit }=0]_{r}\left(\neg K w_{e}(\right.$ bit $=$ $0) \wedge K_{e} K w_{r}($ bit $\left.=0)\right)$.

Now compare model $\mathscr{N}$ above with the following model $\mathscr{N}^{\prime}$, which is bisimilar in the standard sense [7] (which we call modally bisimilar or m-bisimilar): 


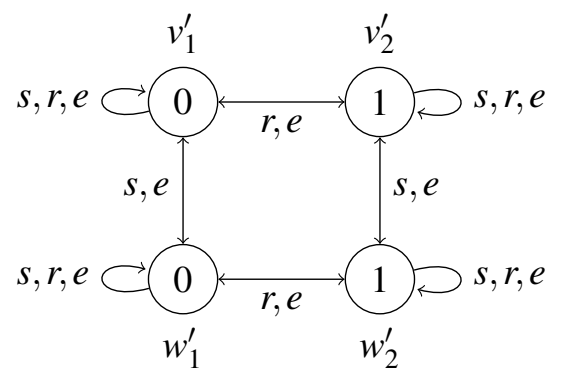

However, this time, after communicating to $r$ the value of the bit, we obtain the updated model $\mathscr{N}_{\left(w_{1}^{\prime}, b i t=0, r\right)}^{\prime}$, which is not bisimilar to $\mathscr{N}^{\prime}$ :

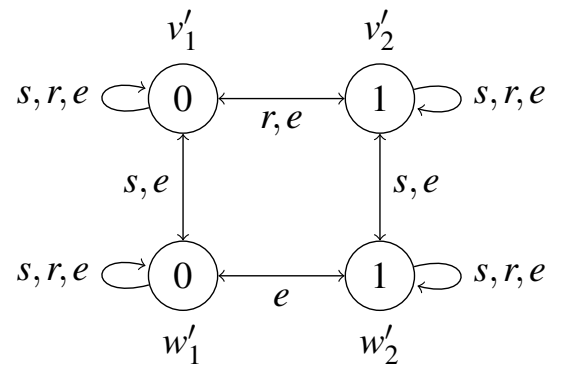

In particular, in $w_{1}^{\prime}$ eavesdropper e does not know that receiver $r$ has learnt the value of the bit: $\left(\mathscr{N}^{\prime}, w_{1}^{\prime}\right)=[\text { bit }=0]_{r} \neg K_{e} K w_{r}($ bit $=0)$. We elaborate on the fact that formulas in GLAL are not preserved under standard modal bisimulations. Specifically, in model $\mathscr{N}^{\prime}$, and differently from $\mathscr{N}$, sender $s$ and eavesdropper $e$ are not able to distinguish between bisimilar states $w_{1}^{\prime}$ and $v_{1}^{\prime}$, which are nonetheless distinct for receiver $r$. We can interpret this feature of $\mathscr{N}^{\prime}$ as formalising the fact that $s$ and e are uncertain as to r's behaviour. Indeed, since these states are indistinguishable for $s$ and e, then they must differ as to the epistemic state of $r$. And this is reflected in the different results of announcing bit $=0$ to $r$. This subtle distinction is reminiscent of the notion of attentive announcements put forward in [8]. We discuss this point in detail in Section 3.

These examples are intended to illustrate the formal features of GLAL to represent global and local communication. In particular, GLAL allows to express local communication that cannot be captured in PAL. In Section 5 we analyse the expressivity of GLAL and provide a formal proof of the fact that it is strictly more expressive than PAL. But first we compare GLAL to related accounts in the literature.

\section{Discussion and Comparison}

In this section we compare our logic to other dynamic epistemic logics, and to accounts of awareness.

Public announcements The logic GLAL can embed public announcement logic [4]. We show that the global announcement modality $[\phi]_{A g}^{+}$for the grand coalition simulates operator $[\phi]$ from PAL. Let us recall the satisfaction clause for $[\phi]$-formulas in PAL:

$$
\left[\left[[\psi] \psi^{\prime}\right]\right]_{\mathscr{M}}=\left\{w \in W \mid \text { if } w \in[[\psi]]_{\mathscr{M}} \text { then } w \in\left[\left[\psi^{\prime}\right]\right]_{\mathscr{M}}\right\}
$$

where the refinement $\mathscr{M}_{\psi}=\left\langle W_{\psi},\left\{R_{\psi, a}\right\}_{a \in A g}, V_{\psi}\right\rangle$ of model $\mathscr{M}$ w.r.t. formula $\psi$ is defined as $(i) W_{\psi}=$ $W \cap[[\psi]]_{\mathscr{M}}$; (ii) for every agent $a \in A g, R_{\psi, a}=R_{a} \cap\left([[\psi]]_{\mathscr{M}} \times[[\psi]] \mathscr{M}\right.$ ); and (iii) for every $p \in A P$,

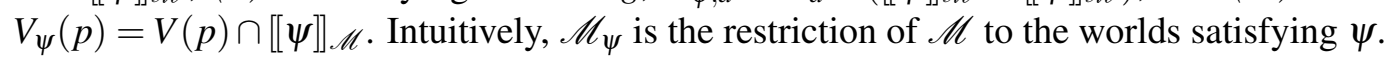


Now consider the recursively defined embedding $\operatorname{tr}: \mathscr{L}_{\text {pal }} \rightarrow \mathscr{L}_{\text {glal }}$ with only non-trivial clause $\operatorname{tr}\left([\phi] \phi^{\prime}\right)=[\operatorname{tr}(\phi)]_{A g}^{+} \operatorname{tr}\left(\phi^{\prime}\right)$. It is then easy to prove that

Proposition 2 For all formulas $\psi$ in $P A L,(\mathscr{M}, w) \models \psi$ iff $(\mathscr{M}, w) \models \operatorname{tr}(\psi)$.

Sketch of Proof. The only non-trivial case is for $\psi=[\phi] \phi^{\prime}$. This follows by inductive hypothesis on $\phi$ and $\phi^{\prime}$, by observing that in $\mathscr{M}_{(w, \phi, A g)}^{+}$, any state $w^{\prime}$ is reachable from $w$ via $R_{A g}^{+C}$ iff $w^{\prime}$ is reachable from $w$ in $\mathscr{M}_{\phi}$.

The following corollary follows immediately from Proposition 2

Corollary 3 For all formulas $\phi, \psi$ in $P A L,(\mathscr{M}, w) \models[\phi]_{A g}^{+} \psi$ iff $(\mathscr{M}, w) \models[\phi] \psi$

In Section 5 we use that public announcement logic can be embedded into GLAL, to demonstrate that GLAL is at least as expressive as PAL.

Private announcements A local announcement is a private announcement to some agents. It will therefore come as no surprise that there is also a strong relation between GLAL and private announcements. Semi-private announcements have been proposed and discussed in in [16, 9, 18, 3]. Specifically, after announcing semi-privately $\phi$ to coalition $A$, all agents in $A$ know that $\phi$ is true, and the agents in $A g \backslash A$ know that all agents in $A$ know whether $\phi$ is true. Now compare this to a local announcement of $\phi$ to coalition $A$, after which all agents in $A$ know that $\phi$ is true, and the agents in $A g \backslash A$ are uncertain between all agents in $A$ knowing that $\phi$ is true, or not having observed the announcement. The distinction between knowing whether and knowing that is not so fundamental here (we could just as well have tweaked the semantics to have knowing whether announcements $[\phi]_{A}^{+}$and $[\phi]_{A}^{-}$.) Whereas the locality of our framework is an essential difference.

The standard way to define the semantics of semi-private announcement is by refinement of the accessibility relation, namely as $R_{a}^{s p}=R_{a}$ for $a \notin A$, whereas $\left.R_{a}^{s p}=R_{a} \cap\left([[\psi]]_{\mathscr{M}}^{2} \cup[[\neg \psi]]_{\mathscr{M}}^{2}\right)\right)$ for $a \in A$. Staying close to our semantics, and using, as for public announcements, the isomorphy of point-generated submodels, semi-private announcements can be interpreted by model refinement $\mathscr{M}_{(w, \psi, a)}^{s p}$ according to which $W^{s p}=W, V^{s p}=V$, and for $a \in A$,

$$
R_{a}^{s p}(v)= \begin{cases}R_{a}(v) \cap[[\psi]]_{\mathscr{M}} & \text { if } v \in R_{A g}^{C}(w) \cap[[\psi]]_{\mathscr{M}} \\ R_{a}(v) \cap[[\neg \psi]]_{\mathscr{M}} & \text { if } \left.v \in R_{A g}^{C}(w) \cap[\neg \psi]\right]_{\mathscr{M}} \\ R_{a}(v) & \text { otherwise }\end{cases}
$$

However, there is no embedding in this case.

Attentive announcements In [8] the authors introduce a logic of attention-based announcements: agents will only process the new information $\phi$ if they are paying attention. Whether they pay attention is handled by a designated set of propositional variables. There is a surprising close relation to our logic GLAL, despite the absence of such attention variables. Consider again Example 2, wherein we modelled the announcement $[$ bit $=0]$ to agent $r$ in state $w_{1}^{\prime}$ of model $\mathscr{N}^{\prime}$. Ignore the role of agent $e$ in the modelling. Although $r$ processes the new information, agent $s$ is uncertain about this fact. Agent $s$ considers that possible, but also considers it possible that $r$ remains uncertain between $b i t=0$ and bit $=1$, namely in states $v_{1}^{\prime}$ and $v_{2}^{\prime}$. Now consider adding an 'attention variable' $h_{r}$ for agent $r$ to the model, as in [8], such that $h_{r}$ is true in $w_{1}^{\prime}$ and $w_{2}^{\prime}$ but false in $v_{1}^{\prime}$ and $v_{2}^{\prime}$. Then (modulo technical details) such an announcement of bit $=0$ to $r$ corresponds to an attention-based announcement of bit $=0$ to $r$ wherein agent $s$ is uncertain whether $r$ is paying attention. The 'technical details' in which the semantics differ 
are that (i) in [8] truly private aspects of announcements are modelled (the believed announcements of [16]), which do not preserve equivalence relations, whereas in our proposal we consider semi-private announcements that preserve equivalence relations; and (ii) our announcements are not necessarily public. But, essentially, an announcement $[\phi]_{A}^{-}$is strictly related to an attention-based announcement of $\phi$ to the agents in $A$, wherein agents not in $A$ are uncertain whether those in $A$ are individually paying attention. On the other hand, an announcement $[\phi]_{A}^{+}$is related to an attention-based announcement of $\phi$ to the agents in $A$, wherein agents not in $A$ are uncertain whether those in $A$ are jointly paying attention. It seems remarkable that in GLAL we can simulate attention of agents without designated variables.

\section{Validities}

In this section we consider notable validities in GLAL, which shed light on the meaning of the global and local announcement operators. Firstly, we observe that after announcing (truthfully) a propositional formula $\phi$ to the agents in $A$, they know $\phi$.

Proposition 4 For every propositional formula $\phi \in \mathscr{L}_{p l}$,

$$
\begin{aligned}
& \models[\phi]_{A}^{-} E_{A} \phi \\
& =[\phi]_{A}^{+} C_{A} \phi
\end{aligned}
$$

According to Proposition 4, if a propositional formula $\phi$ is announced locally, then all agents involved in the announcement jointly know $\phi$; while if $\phi$ is announced globally, then it also becomes common knowledge. Proposition 4 does not hold for general formulas $\phi \in \mathscr{L}_{\text {glal }}$. As a counterexample, consider Moore's formula $p \wedge \neg K_{a} p$.

We anticipate that, as a consequence of Theorem 6 below, differently from PAL there is no set of validities in GLAL to rewrite any announcement in terms of simpler formulas. Nonetheless, the following formulas are validities in GLAL:

$$
\begin{aligned}
{[\phi]_{A}^{-} p } & \leftrightarrow \phi \rightarrow p \\
{[\phi]_{A}^{-} \neg \psi } & \leftrightarrow \phi \rightarrow \neg[\phi]_{A}^{-} \psi \\
{[\phi]_{A}^{-}\left(\psi \wedge \psi^{\prime}\right) } & \leftrightarrow[\phi]_{A}^{-} \psi \wedge[\phi]_{A}^{-} \psi^{\prime}
\end{aligned}
$$

and similarly for $[\phi]_{A}^{+}$. Thus, both announcement operators commute with propositional connective.

Moreover, epistemic operators and nested announcements commute with the announcement operators if they refer to the same coalition of agents.

Proposition 5 The following are GLAL validities:

$$
\begin{aligned}
{[\phi]_{A}^{-} E_{A} \psi } & \leftrightarrow \phi \rightarrow E_{A}[\phi]_{A}^{-} \psi \\
{[\phi]_{A}^{-}\left[\phi^{\prime}\right]_{A}^{-} \psi } & \leftrightarrow\left[\phi \wedge[\phi]_{A}^{-} \phi^{\prime}\right]_{A}^{-} \psi \\
{[\phi]_{A}^{+}\left[\phi^{\prime}\right]_{A}^{+} \psi } & \leftrightarrow\left[\phi \wedge[\phi]_{A}^{+} \phi^{\prime}\right]_{A}^{+} \psi
\end{aligned}
$$

On the other hand, formulas (3)-(5) do not hold if the coalition appearing in the announcement operator is different from the coalition appearing in the epistemic operator.

Given that operators $[\phi]_{A}^{-}$and $[\phi]_{A}^{+}$are not reducible, it is of interest to investigate what kind of modalities they are, specifically what modal principles their semantics validates. First, it is easy to see 
that both axiom $\mathbf{K}$ and rule $\mathbf{N e c}$ of necessitation are valid:

$$
\begin{array}{r}
{[\phi]_{A}^{-}\left(\psi \rightarrow \psi^{\prime}\right) \rightarrow\left([\phi]_{A}^{-} \psi \rightarrow[\phi]_{A}^{-} \psi^{\prime}\right)} \\
\psi \Rightarrow[\phi]_{A}^{-} \psi
\end{array}
$$

and the same validities hold for operator $[\phi]_{A}^{+}$.

On the other hand, all axioms $\mathbf{T}, \mathbf{4}$ and $\mathbf{B}$ fail. As regards $\mathbf{T}$, if $\phi$ is false, then $[\phi]_{a} \psi$ holds for any formula $\psi$, but it does not follow that $\psi$ holds whenever it is false itself. As to 4, notice that in the muddy children puzzle a child not stepping forward is tantamount to globally stating that she does not know whether she is muddy, or $\left[n_{-} s t\right]:=\left[\bigwedge_{i \in A g} \neg K w_{i} m_{i}\right]_{A g}^{+}$. Hence, after the father's announcement, in state $(1,1,0)$ we have that no child knows whether she is muddy after the first round, that is, $(1,1,0) \models$ $[$ no_st $] \bigwedge_{i \in A g} \neg K w_{i} m_{i}$. However, at the second round all muddy children know that they are muddy: $(1,1,0) \models[$ no_st $][$ no_st $] \bigwedge_{i \in A g}\left(m_{i} \rightarrow K w_{i} m_{i}\right)$. In particular, $(1,1,0) \not \models[$ no_st $][$ no_st $] \bigwedge_{i \in A g} \neg K w_{i} m_{i}$, thus invalidating 4. As regards $B$, we can show that it fails by considering Moore's formula $p \wedge \neg K_{a} p$ and a pointed model $(\mathscr{M}, w)$ such that $(\mathscr{M}, w) \models p \wedge \neg K_{a} p$ but $(\mathscr{M}, w) \not \models[p]_{a}\langle p\rangle_{a}\left(p \wedge \neg K_{a} p\right)$.

\section{GLAL, PAL, and Epistemic Logic}

The main result in this section is that GLAL, differently from PAL, is not reducible to epistemic logic, but rather strictly more expressive than both. In Section 3 we proved that GLAL is at least as expressive as public announcement logic. Next we prove that GLAL is strictly more expressive, in the sense that some formulas in GLAL are not equivalent to any epistemic formula. Since epistemic logic and PAL are equally expressive [4], it immediately follows that GLAL is strictly more expressive than PAL as well.

Theorem 6 GLAL is strictly more expressive than epistemic logic with common knowledge.

Sketch of Proof. We prove this result by providing two modally bisimilar models, that therefore satisfy the same epistemic formulas, but satisfy different formulas in GLAL. Consider models $\mathscr{N}$ and $\mathscr{N}^{\prime}$ in Example 2 and define a relation $B$ such that $B\left(w_{i}, w_{i}^{\prime}\right)$ and $B\left(w_{i}, v_{i}^{\prime}\right)$, for $i \in\{1,2\}$. It is easy to check that the $B$ is a modal bisimulation between pointed models $\left(\mathscr{N}, w_{1}\right)$ and $\left(\mathscr{N}^{\prime}, w_{1}^{\prime}\right)$. In particular, the same epistemic formulas are satisfied at states $w_{1}$ and $w_{1}^{\prime}$. However, as we noticed in Example 2, for $\phi::=[\text { bit }=0]_{r} K_{e} K w_{r}($ bit $=0)$, we can check $\left(\mathscr{N}, w_{1}\right) \models \phi$; while $\left(\mathscr{N}^{\prime}, w_{1}^{\prime}\right) \not \models \phi$. In particular, there is no epistemic formula in $\mathscr{L}_{e l}$ that is equivalent to $\phi$.

By Theorem 6 and the equi-expressivity of epistemic logic and PAL [4], we immediately obtain the following corollary.

\section{Corollary 7 GLAL is strictly more expressive than PAL.}

By Example 2 and the proof of Theorem 6 not even announcements to single agents are reducible to epistemic formulas. Also, the same proof points out that a more robust notion of bisimulation is needed to preserve formulas in GLAL. We explore such a notion in the next section.

\section{Bisimulations}

In this section we investigate a stronger notion of bisimulation capable of preserving the meaning of GLAL formulas as well. Firstly, for any set $A \subseteq A g$ of agents, and model $\mathscr{M}=\left\langle W,\left\{R_{a}\right\}_{a \in A g}, V\right\rangle$, we define $R_{A}(w, v)$ as: $R_{a}(w, v)$ iff $a \in A$, that is, $R_{A}(w, v)$ holds iff $R_{a}(w, v)$ holds for exactly the agents $a \in A$. 
Definition 4 ( \pm -Simulation) Given models $\mathscr{M}$ and $\mathscr{M}^{\prime}, a \pm$-simulation is a relation $\mathbf{S} \subseteq W \times W^{\prime}$ such that $\mathbf{S}\left(w, w^{\prime}\right)$ implies

Atoms $w \in V(p)$ iff $w^{\prime} \in V^{\prime}(p)$, for every $p \in A P$;

Forth for every $A \subseteq A g$ and $v \in W$, if $R_{A}(w, v)$ then for some $v^{\prime} \in W^{\prime}, R_{A}^{\prime}\left(w^{\prime}, v^{\prime}\right)$ and $\mathbf{S}\left(v, v^{\prime}\right)$;

Reach if $\mathbf{S}\left(v, v^{\prime}\right)$ then for every $a \in A g, R_{a}(w, v)$ iff $R_{a}^{\prime}\left(w^{\prime}, v^{\prime}\right)$.

Besides conditions Atoms and Forth, Reach is required to preserve the interpretation of formulas when refinements are considered. A \pm -bisimulation is a relation $\mathbf{B} \subseteq W \times W^{\prime}$ such that both $\mathbf{B}$ and $\mathbf{B}^{-1}=\left\{\left(w^{\prime}, w\right) \mid \mathbf{B}\left(w, w^{\prime}\right)\right\}$ are simulations. Two worlds $w, w^{\prime}$ are \pm -bisimilar, or $w \rightleftharpoons w^{\prime}$, iff $\mathbf{B}\left(w, w^{\prime}\right)$ holds for some \pm -bisimulation $\mathbf{B}$. Further, two models $\mathscr{M}$ and $\mathscr{M}^{\prime}$ are \pm -bisimilar, or $\mathscr{M} \rightleftharpoons \mathscr{M}^{\prime}$, iff $(i)$ for every $w \in \mathscr{M}, w \rightleftharpoons w^{\prime}$ for some $w^{\prime} \in \mathscr{M}^{\prime}$; and (ii) for every $w^{\prime} \in \mathscr{M}^{\prime}, w \rightleftharpoons w^{\prime}$ for some $w \in \mathscr{M}$. We write $(\mathscr{M}, w) \rightleftharpoons\left(\mathscr{M}^{\prime}, w^{\prime}\right)$ to state that $w$ and $w^{\prime}$ are \pm -bisimilar in \pm -bisimilar $\mathscr{M}, \mathscr{M}^{\prime}$.

Clearly, by Def. $4 \pm$-bisimilarity implies $m$-bisimilarity. However, the opposite implication does not hold. Notably, the $m$-bisimilar models $\mathscr{N}$ and $\mathscr{N}^{\prime}$ in Example 2 are not \pm -bisimilar according to Def.4 We now show that global and local announcements preserve bisimilarity.

Theorem 8 Suppose that $(\mathscr{M}, s) \rightleftharpoons\left(\mathscr{M}^{\prime}, s^{\prime}\right)$. Then, for every formula $\psi$ in $G L A L$

$$
(\mathscr{M}, s) \models \psi \quad \text { iff } \quad\left(\mathscr{M}^{\prime}, s^{\prime}\right) \models \psi
$$

In particular, if $\psi=[\theta]_{A}^{-} \theta^{\prime}$ (resp. $\left.\psi=[\theta]_{A}^{+} \theta^{\prime}\right)$ then $\mathscr{M}_{(s, \theta, A)}^{-} \rightleftharpoons \mathscr{M}_{\left(s^{\prime}, \theta, A\right)}^{\prime-}$ and $\mathscr{M}_{(s, \theta, A)}^{+} \rightleftharpoons \mathscr{M}_{\left(s^{\prime}, \theta, A\right)}^{\prime+}$.

Discussion: Distributed knowledge There is a close relation between our notion of \pm -bisimulation and the notion known in the literature as collective bisimulation [22, 23]. The (only) difference is that in Def. 4 we require $R_{A}$ to be exact $\left((w, v) \in R_{A}\right.$ iff: $(w, v) \in R_{a}$ for $a \in A$ and $(w, v) \notin R_{a}$ for $\left.a \notin A\right)$ whereas in collective bisimulation $R_{A}$ is taken inclusively $\left((w, v) \in R_{A}\right.$ iff: $(w, v) \in R_{a}$ for $\left.a \in A\right)$. Clearly, \pm -bisimilar models are also collectively bisimilar, so \pm -bisimilarity also implies equivalence in the logic of distributed knowledge. However, the other way round may not hold. As an example, consider again models $\mathscr{N}$ and $\mathscr{N}^{\prime}$ in Example 2. These are collectively bisimilar, as well as $m$-bisimilar. However, they are not \pm -bisimilar. In particular, it is easy to see that $\left(\mathscr{N}, w_{1}\right) \models[b i t=0]_{r} K_{e} D_{r, e}($ bit $=0)$; whereas $\left(\mathscr{N}^{\prime}, w_{1}^{\prime}\right) \not \models[b i t=0]_{r} K_{e} D_{r, e}(b i t=0)$. As a consequence, we obtain the following result:

Theorem 9 Epistemic logic with distributed knowledge is not as expressive as GLAL.

This begs the question as to how logics with distributed knowledge relate to GLAL and, for example, what their relative expressivity is. For instance, we do not know whether GLAL is strictly more expressive or the two logics are uncomparable.

\section{Model Checking and Satisfaction}

As part of the analysis of the formal properties of GLAL, we investigate the model checking and satisfiability problems, defined as follows.

\section{Definition 5 (Model Checking and Satisfiability)}

- Model Checking Problem: Given a finite model $\mathscr{M}$, state $w$ in $\mathscr{M}$, and formula $\phi$ in GLAL, determine whether $(\mathscr{M}, w)=\phi$.

- Satisfiability Problem: Given a formula $\phi$ in $G L A L$, determine whether $(\mathscr{M}, w) \models \phi$ for some model $\mathscr{M}$ and state $w$ in $\mathscr{M}$. 
The model checking problem is tantamount to determine whether $w \in[[\phi]] \mathscr{M}$, hence it depends crucially on the complexity of computing the satisfaction set $[[\phi]] \mathscr{M}$, as membership is supposed to be computable in polynomial time.

Theorem 10 The model checking problem for GLAL is PTIME-complete.

A consequence of Theorem 10 is that model checking GLAL is no more computationally complex than the verification of epistemic logic. Hence, the enhanced expressivity of GLAL comes at no extra computational cost from a verificational perspective.

Theorem 11 The satisfiability problem for GLAL is decidable.

In Theorem 11 the decidability of GLAL is proved similarly to the decidability of PAL. The difference is merely in the amount of transitions due to announcements. Whereas in PAL announcements $[\phi]$ are functional, in GLAL announcements $[\phi]_{A}^{+}$and $[\phi]_{A}^{-}$are branching (i.e., for each $A \subseteq A g$, and for global and local announcements, we may need different transitions). In that sense the decidability proof is more akin to that of action model logic, wherein non-deterministic actions also cause branching.

\section{Conclusions}

We introduced a unified account to formalise both global and local announcements in GLAL, a strictly more expressive extension of public announcement logic. The key feature of the semantics of GLAL is that the refinement of the indistinguishability relations is defined in the same way for public and private announcement, i.e., as the restriction of the equivalence classes to the worlds satisfying (or not) the given announcement. The crucial difference between global and local announcements is the domain of application of such updates: worlds accessible in one step or all worlds epistemically reachable, respectively. In Example 1 and 2 we showed how these formal notions capture our intuitions about global and local announcements.

In future work we envisage several extensions. Firstly, since differently from PAL, announcements are not necessarily broadcast to all agents (so that only one such announcement can be broadcast at each given time), we can envisage global and local announcements communicated simultaneously and introduce formulas $\left([\phi]_{A} \circ\left[\phi^{\prime}\right]_{B}\right) \psi$ with the intended meaning that if $\phi$ is (truthfully) announced to coalition $A$ and simultaneously $\phi^{\prime}$ is announced to coalition $B$, then $\psi$ holds. This is of interest to model synchronous communication. Particular care is to be taken in defining the semantics of operator $[\phi]_{A} \circ\left[\phi^{\prime}\right]_{B}$ whenever the intersection of coalitions $A$ and $B$ is non-empty.

Secondly, as the receiver of the announcement can be a subset $A \subseteq A g$ of the set of all agents, we can think that the announcement originates from some other coalition $B$ and introduce GLAL operators $[\phi]_{B, A}$ indexed to both $A$ and $B$. Such an extension would provide a finer-grained analysis of scenarios such as communication and security protocols.

\section{Acknowledgements}

We thank the TARK reviewers for the comments. The research described in this paper was supported by the French ANR JCJC Project SVeDaS (ANR-16-CE40-0021) and the ERC project EPS 313360. Hans van Ditmarsch is also affiliated to IMSc, Chennai. 


\section{References}

[1] C. Areces, R. Fervari \& G. Hoffmann (2012): Moving Arrows and Four Model Checking Results. In: Proc. of 19th WoLLIC, Springer, pp. 142-153, doi:10.1007/978-3-642-32621-9_11. LNCS 7456.

[2] G. Aucher, P. Balbiani, L. Fariñas del Cerro \& A. Herzig (2009): Global and Local Graph Modifiers. ENTCS 231, pp. 293-307, doi:10.1016/j.entcs.2009.02.042.

[3] A. Baltag, H. van Ditmarsch \& L.S. Moss (2008): Epistemic Logic and Information Update. In: Handbook on the Philosophy of Information, Elsevier, pp. 361-456, doi:10.1016/B978-0-444-51726-5.50015-7.

[4] A. Baltag, L. Moss \& S. Solecki (1998): The Logic of Public Announcements, Common Knowledge, and Private Suspicions. In: Proc. of 7th TARK, Morgan K., pp. 43-56, doi:10.1007/978-3-319-20451-2_38

[5] J. van Benthem (2005): An Essay on Sabotage and Obstruction. In: Mechanizing Mathematical Reasoning, LNCS 2605, Springer, pp. 268-276, doi:10.1007/978-3-540-32254-2_16

[6] J. van Benthem (2011): Logical Dynamics of Information and Interaction. Cambridge University Press, doi $10.1017 /$ cbo9780511974533.002.

[7] P. Blackburn, M. de Rijke \& Y. Venema (2001): Modal Logic. Cambridge University Press, doi $10.1017 /$ cbo9781107050884

[8] T. Bolander, H. Ditmarsch, A. Herzig, E. Lorini, P. Pardo \& F. Schwarzentruber (2016): Announcements to Attentive Agents. J. of Logic, Lang. and Inf. 25(1), pp. 1-35, doi 10.1007/s10849-015-9234-3

[9] H. van Ditmarsch (2000): Knowledge games. ILLC Dissertation Series DS-2000-06.

[10] H. van Ditmarsch, J. Halpern, W. van der Hoek \& B. Kooi, editors (2015): Handbook of epistemic logic. College Publications.

[11] H. van Ditmarsch, W. van der Hoek \& B. Kooi (2004): Public Announcements and Belief Expansion. In: Advances in Modal Logic 5, pp. 62-73.

[12] Hans van Ditmarsch \& Barteld Kooi (2015): One Hundred Prisoners and a Light Bulb. Copernicus, doi $10.1007 / 978-3-319-16694-0.9$.

[13] H.P. van Ditmarsch, W. van der Hoek \& B.P. Kooi (2007): Dynamic Epistemic Logic. Synthese Library 337, Springer, doi $10.1007 / 978-1-4020-5839-4$

[14] R. Fagin, J.Y. Halpern, Y. Moses \& M.Y. Vardi (1995): Reasoning About Knowledge. The MIT Press.

[15] R. Fervari (2014): Relation-Changing Modal Logics. Ph.D. thesis, Universidad Nacional de Córdoba, Argentina.

[16] J.D. Gerbrandy \& W. Groeneveld (1997): Reasoning about Information Change. JoLLI 6, pp. 147-169, doi $10.1023 / \mathrm{A}: 1008222603071$.

[17] J.Y. Halpern \& Y. Moses (1992): A guide to completeness and complexity for modal logics of knowledge and belief. J. of AI 54, pp. 319-379, doi:10.1016/0004-3702(92)90049-4.

[18] W. van der Hoek \& M. Pauly (2006): Modal Logic for Games and Information. In: The Handbook of Modal Logic, Elsevier, pp. 1180-1152, doi:10.1016/s1570-2464(07)80023-1.

[19] B. Kooi \& B. Renne (2011): Arrow Update Logic. Review of Symbolic Logic 4(4), pp. 536-559, doi $10.1017 /$ S1755020311000189

[20] J.Ch. Meyer \& W. van der Hoek (1995): Epistemic Logic for AI and Computer Science. Cambridge University Press, doi:10.1017/CBO9780511569852.

[21] J.A. Plaza (1989): Logics of Public Communications. In: Proc. of the 4th ISMIS, Oak Ridge National Laboratory, pp. 201-216, doi $10.1007 / \mathrm{s} 11229-007-9168-7$

[22] F. Roelofsen (2007): Distributed knowledge. JANCL 17(2), pp. 255-273, doi 10.3166/jancl.17.255-273.

[23] Y.N. Wáng \& T. Ågotnes (2013): Public announcement logic with distributed knowledge: expressivity, completeness and complexity. Synthese 190, pp. 135-162, doi:10.1007/s11229-012-0243-3. 


\section{Appendix with Proofs}

Proof. (Proposition 2) The only non-trivial case is for $\psi=[\phi] \phi^{\prime}$. In particular, we show that for every $w \in W$, refinement $\mathscr{M}_{(w, \phi, A g)}^{+}$satisfies the same formulas in PAL as refinement $\mathscr{M}_{\phi}$. The key remark here is that worlds that are not reachable from $w$ via relation $R_{A g}^{C}$ do not account for the satisfaction of PAL formulas at $w$. Specifically, in refinement $\mathscr{M}_{(w, \phi, A g)}^{+}$, any state $w^{\prime}$ is reachable from $w$ via $R_{A g}^{+C}$ iff $w^{\prime}$ is reachable from $w$ in $\mathscr{M}_{\phi}$. Also, in both refinements the indistinguishability relations and assignments are restricted to $[[\phi]]_{\mathscr{M}}$. As a result, the two models satisfy the same $[\phi]$-formulas at $w$.

Proof. (Proposition 4) We prove (1) for a propositional formula $\phi$. Suppose that $(\mathscr{M}, w) \models \phi$ but $\left(\mathscr{M}_{(w, \phi, A)}^{-}, w\right) \not \models E_{A} \phi$ to obtain a contradiction, that is, $\left(\mathscr{M}_{(w, \phi, A)}^{-}, w^{\prime}\right) \not \models \phi$ for some $a \in A$ and $w^{\prime} \in R_{a}^{-}(w)$. In particular, this means that $\left(\mathscr{M}, w^{\prime}\right) \not \neq \phi$, as $\phi$ is propositional. Hence, $w^{\prime} \neq w$ (as $\phi$ is true in $w$ ) and $w^{\prime} \in R_{a}(w) \supseteq R_{a}^{-}(w)$. But then $\left.w^{\prime} \notin R_{a}(w) \cap[\phi]\right]$, against the hypothesis that $R_{a}^{-}\left(w, w^{\prime}\right)$. Therefore, it is the case that $\left(\mathscr{M}_{(w, \phi, A)}^{-}, w\right) \models E_{A} \phi$. The proof for 2 follows a similar line.

Proof. (Proposition 5) We prove (4). Suppose that $(\mathscr{M}, w) \models[\phi]_{A}^{-}\left[\phi^{\prime}\right]_{A}^{-} \psi$, that is, if $(\mathscr{M}, w) \models \phi$ and $\left(\mathscr{M}_{(w, \phi, A)}^{-}, w\right) \models \phi^{\prime}$, then $\left(\left(\mathscr{M}_{(w, \phi, A)}^{-}\right)_{\left(w, \phi^{\prime}, A\right)}^{-}, w\right) \models \psi$. We have to show that this is equivalent to $(\mathscr{M}, w) \models\left[\phi \wedge[\phi]_{A}^{-} \phi^{\prime}\right]_{A}^{-} \psi$, that is, if $(\mathscr{M}, w) \models \phi$ and $\left(\mathscr{M}_{(w, \phi, A)}^{-}, w\right) \models \phi^{\prime}$, then $\left(\mathscr{M}_{\left(w, \phi \wedge[\phi]_{A}^{-} \psi^{\prime}, A\right)}^{-}, w\right) \models$ $\psi$. Hence, it is enough to prove that $\left(\left(\mathscr{M}_{(w, \phi, A)}^{-}\right)_{\left(w, \phi^{\prime}, A\right)}^{-}, w\right) \models \psi$ iff $\left(\mathscr{M}_{\left(w, \phi \wedge[\phi]_{A}^{-} \psi^{\prime}, A\right)}^{-}, w\right) \models \psi$. In particular, refinements $\left(\mathscr{M}_{(w, \phi, A)}^{-}\right)_{\left(w, \phi^{\prime}, A\right)}^{-}$and $\mathscr{M}_{\left(w, \phi \wedge[\phi]_{A}^{-} \psi^{\prime}, A\right)}^{-}$are identical. To see this we remark that in refinement $\left(\mathscr{M}_{(w, \phi, A)}^{-}\right)_{\left(w, \phi^{\prime}, A\right)}^{-}$, for every $a \in A$,

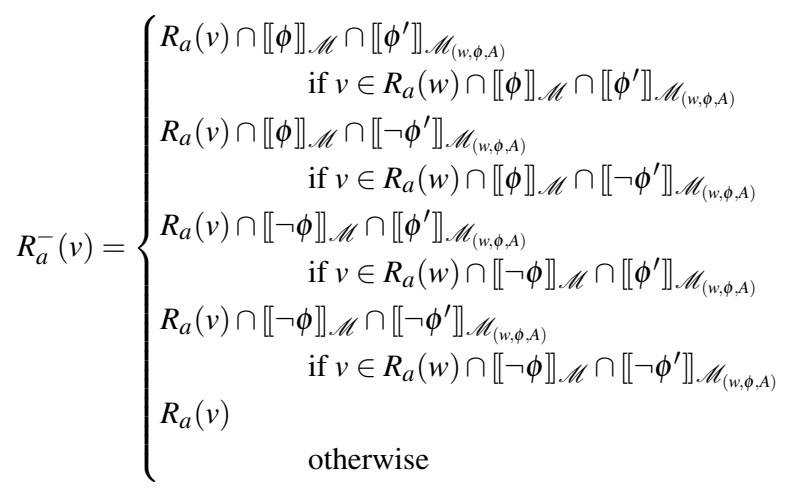

which is tantamount to the following in model $\mathscr{M}_{\left(w, \phi \wedge[\phi]_{A}^{-} \phi^{\prime}, A\right)}^{-}$:

$$
R_{a}^{-}(v)= \begin{cases}R_{a}(v) \cap \llbracket \phi \wedge[\phi]_{A} \phi^{\prime} \rrbracket \mathscr{M} & \text { if } \left.v \in R_{a}(w) \cap \llbracket \phi \wedge[\phi]_{A} \phi^{\prime} \rrbracket\right]_{\mathscr{M}} \\ R_{a}(v) \cap \llbracket \neg\left(\phi \wedge[\phi]_{A} \phi^{\prime}\right) \rrbracket_{\mathscr{M}} & \text { if } v \in R_{a}(w) \cap \llbracket \neg\left(\phi \wedge[\phi]_{A} \phi^{\prime}\right) \rrbracket_{\mathscr{M}} \\ R_{a}(v) & \text { otherwise }\end{cases}
$$

Hence, the two models are identical and (4) holds.

Proof. (Theorem 8 ) The proof is by induction on the structure of formula $\psi$. The base case for atomic propositions and the inductive cases for propositional connectives and epistemic modalities are immediate, as \pm -bisimulations are $m$-bisimulations in particular. So suppose that $\psi=[\theta]_{A}^{-} \theta^{\prime}$, and assume the induction hypothesis for both $\theta$ and $\theta^{\prime}$. We can guarantee this by assuming that the complexity of $\theta$ is lower than that of $\theta^{\prime}$. This, in turn, can be achieved for instance by allowing only update formulas of the form $[\theta]_{A}^{-}\left(\left(\theta^{\prime} \wedge \theta\right) \vee\left(\theta^{\prime} \wedge \neg \theta\right)\right)$. 
Next, we consider the local refinements $\mathscr{M}_{(s, \theta, A)}^{-}$and $\mathscr{M}_{\left(s^{\prime}, \theta, A\right)}^{\prime-}$, and prove Atoms, Forth and Reach from left to right, the other direction being symmetric. We assume a bisimulation $\mathbf{B} \subseteq W \times W^{\prime}$ and show that $\mathbf{B}$ is a bisimulation between $\mathscr{M}_{(s, \theta, A)}^{-}$and $\mathscr{M}_{\left(s^{\prime}, \theta, A\right)}^{-}$as well, such that $\mathbf{B}\left(s, s^{\prime}\right)$ holds. Firstly, for every $w \in W^{-}=W$ and $w^{\prime} \in W^{\prime-}=W^{\prime}$, if $\mathbf{B}\left(w, w^{\prime}\right)$ then $w \in V^{-}(p)$ iff $w \in V(p)$ iff $w^{\prime} \in V^{\prime}(p)$ iff $w^{\prime} \in V^{\prime-}(p)$, which shows that Atoms is satisfied.

As regards Forth, let us write $R_{A}^{E}(s)$ for $\bigcup_{a \in A} R_{a}(s)$. Then, we distinguish the case whether $w \in R_{A}^{E}(s)$ or $w \notin R_{A}^{E}(s)$. First, if $\mathbf{B}\left(w, w^{\prime}\right)$ and $w \notin R_{A}^{E}(s)$, then for every set $B \subseteq A g$ of agents and $v \in W, R_{B}^{-}(w, v)$ iff $R_{B}(w, v)$. Moreover, since $\mathbf{B}\left(s, s^{\prime}\right), w^{\prime} \notin R_{A}^{\prime E}\left(s^{\prime}\right)$ by $\mathbf{R e a c h}$, and therefore for some $v^{\prime} \in W^{\prime}, \mathbf{B}\left(v, v^{\prime}\right)$ and $R_{B}^{\prime}\left(w^{\prime}, v^{\prime}\right)$ implies $R_{B}^{\prime-}\left(w^{\prime}, v^{\prime}\right)$.

Now consider $w \in R_{A}^{E}(s)$ (and therefore $w^{\prime} \in R_{A}^{E}\left(s^{\prime}\right)$ by Reach). We distinguish two cases: (i) $(\mathscr{M}, w) \models \theta$ iff $(M, v) \models \theta$ (i.e., $(\mathscr{M}, w)$ and $(\mathscr{M}, v)$ agree on $\theta)$. By the definition of refinement, we then have that $R_{B}^{-}(w, v)$ iff $R_{B}(w, v)$. Since $\mathbf{B}$ is a bisimulation, we then also have $R_{B}^{\prime}\left(w^{\prime}, v^{\prime}\right)$ for some $v^{\prime} \in W^{\prime}$. Moreover, by induction hypothesis, $\left(\mathscr{M}^{\prime}, w^{\prime}\right)$ and $\left(\mathscr{M}^{\prime}, v^{\prime}\right)$ agree on $\theta$ as well. So we have $R_{B}^{\prime-}\left(w^{\prime}, v^{\prime}\right)$.

Now assume $(i i)(\mathscr{M}, w) \models \theta$ iff $(\mathscr{M}, v) \not \models \theta$ (i.e., $(\mathscr{M}, w)$ and $(\mathscr{M}, v)$ disagree on $\theta)$. Suppose that $R_{B}^{-}(w, v)$. By the definition of refinement, we have $R_{B}(w, v)$, where $B$ is a set for which $C \subseteq B \subseteq(A \cup C)$, that is, all agents in $A \cap B$ have learned that $w$ and $v$ are different. But then we also have $R_{B}^{\prime}\left(w^{\prime}, v^{\prime}\right)$ for some $v^{\prime} \in W^{\prime}$, and again by the definition of refinement, $R_{B}^{\prime-}\left(w^{\prime}, v^{\prime}\right)$.

Finally, condition Reach holds for $\mathscr{M}_{(s, \theta, A)}^{-}$and $\mathscr{M}_{\left(s^{\prime}, \theta, A\right)}^{-}$, as if $\mathbf{B}\left(w, w^{\prime}\right), \mathbf{B}\left(v, v^{\prime}\right)$, and $w \in R_{A}^{E}(s)$, then for every $b \in A g, R_{b}(w, v)$ implies that either $b \notin A$ or $w$ and $v$ agree on the interpretation of $\theta$ in $\mathscr{M}$. By induction hypothesis $w^{\prime}$ and $v^{\prime}$ agree as well on $\theta$, and in particular $R_{b}^{\prime}\left(w^{\prime}, v^{\prime}\right)$.

For the global refinement $\mathscr{M}_{(s, \psi, A)}^{+}$, the proof is similar, but instead of equivalence class $R_{A}^{E}(w)$, we consider $R_{A}^{C}(w)$.
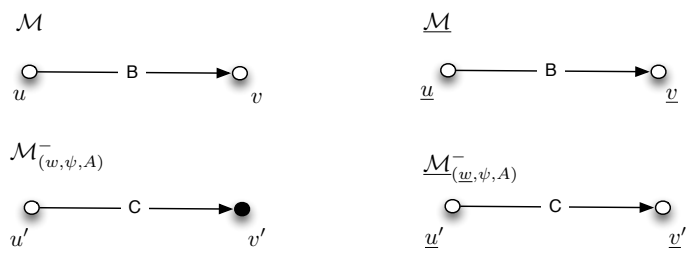

Figure 1: Scheme for the proof of Theorem 8

To conclude the proof, for the case of announcement formulas $\psi=[\theta]_{A}^{-} \theta^{\prime},(\mathscr{M}, w) \models \psi$ iff $(\mathscr{M}, w) \models$ $\theta$ implies $\left.\left(\mathscr{M}_{(w, \theta, A)}^{-}, w\right) \models \theta^{\prime}\right)$. If $(\mathscr{M}, w) \not \neq \theta$, then also $\left(\mathscr{M}^{\prime}, w^{\prime}\right) \not \neq \theta$, so $\left(\mathscr{M}^{\prime}, w^{\prime}\right) \models \psi$. Then, suppose that $(\mathscr{M}, w) \models \theta$. We then have $\left(\mathscr{M}_{(w, \theta, A)}^{-}, w\right) \models \theta^{\prime}$ and, since $\left(\mathscr{M}_{(w, \theta, A)}^{-}, w\right) \leftrightharpoons\left(\mathscr{M}_{\left(w^{\prime}, \theta, A\right)}^{-}, w^{\prime}\right)$ by the proof above, by the induction hypothesis we obtain $\left(\mathscr{M}_{\left(w^{\prime}, \theta, A\right)}^{-}, w^{\prime}\right) \models \theta^{\prime}$, and therefore $\left(\mathscr{M}^{\prime}, w^{\prime}\right) \models \varphi$. The case for $\psi=[\theta]_{A}^{+} \theta^{\prime}$ is similar.

Proof. (Theorem 10 The PTIME-hardness of model checking GLAL follows from the PTIMEhardness of model checking epistemic logic and the fact that the latter is subsumed by the former [20].

As regards membership in PTIME, epistemic formulas can be checked in polynomial time. Further, model refinements $\mathscr{M}_{(w, \psi, A)}^{+}$and $\mathscr{M}_{(w, \psi, A)}^{-}$, for world $w$, formula $\psi$, and coalition $A$, can be computed in polynomial time in the size $|\mathscr{M}|$ of the model given as $|W|+\sum_{a \in A g}\left|R_{a}\right|$. By combining the two procedures we can obtain an algorithm that runs in polynomial time. 
Sketch of Proof. (Theorem 11) To prove the decidability of GLAL we describe a decision procedure inspired by [17]. There, given an epistemic formula $\phi \in \mathscr{L}_{e l}$, the authors provide an algorithm to build a (purely epistemic) model $M=\left(S, \pi, \mathscr{K}_{1}, \ldots, \mathscr{K}_{n}\right)$ in exponential time, in which $(i)$ each state $s \in S$ is a maximal propositional tableau on the set $\operatorname{Sub}_{C}^{+}(\phi)$ of subformulas of $\phi$; ; (ii) $p \in \pi(s)$ iff $p \in s$; (iii) $\mathscr{K}_{a}(s, t)$ iff $\left\{K_{a} \psi \mid K_{a} \psi \in s\right\}=\left\{K_{a} \psi \mid K_{a} \psi \in t\right\}$. In particular, if $\phi$ if satisfiable (in some epistemic model), then $M$ is a model for $\phi$.

Now, based on the above, we describe a procedure to build a (possibly empty) model for a formula $\phi \in \mathscr{L}_{\text {glal }}$. Firstly, by interpreting each announcement subformula in $\psi$ as a new proposition, $\phi$ can be seen as a purely epistemic formula, so that the procedure in [17] applies. Then, we obtain an epistemic model $M$. Further, starting with $M$ we build a GLAL model for $\phi$. Specifically, given $M$ and copies $s_{1}^{\prime}, \ldots, s_{n}^{\prime}$ of states $s_{1}, \ldots, s_{n}$ in $M$, consider a new model $M^{\prime}=\left(S^{\prime}, \pi, \mathscr{K}_{1}^{\prime}, \ldots, \mathscr{K}_{n}^{\prime}\right)$ such that $(i) S^{\prime}=$ $S \cup\left\{s_{1}^{\prime}, \ldots, s_{n}^{\prime}\right\}$; and (ii) for $s, t \in S, \mathscr{K}_{a}^{\prime}(s, t)$ iff $\mathscr{K}_{a}(s, t)$, while for $t \in S, \mathscr{K}_{a}^{\prime}\left(s^{\prime}, t\right)$ and $\mathscr{K}_{a}^{\prime}\left(t, s^{\prime}\right)$ only if $\mathscr{K}_{a}(s, t)$ and $\mathscr{K}_{a}(t, s)$. Notice that all such $M^{\prime}$ are $m$-bisimilar to the original $M$. However, they are not necessarily \pm -bisimilar to $M$, and therefore we need to consider all such $M^{\prime}$ up to \pm -bisimulation when checking for satisfiability. In particular, these are in finite number as $S$ is finite.

Finally, we define a tableau for GLAL as a tuple $T=\left\langle\left\{M_{i}\right\}_{i \in I},\left\{R_{(s, \phi, A)}^{-}\right\},\left\{R_{(s, \psi, A)}^{+}\right\}\right\rangle$, for $s \in S, \psi \in$ $\operatorname{Sub}_{C}^{+}(\phi), A \subseteq A g$, such that

- all $M_{i}=\left\langle S, \pi, \mathscr{K}_{1}, \ldots, \mathscr{K}_{n}\right\rangle$ are epistemic models, all defined on a single set $S$ of states, as described above;

- each $R_{(s, \psi, A)}^{-}$is a relation on epistemic models such that $R_{(s, \psi, A)}^{-}\left(M, M^{\prime}\right)$ and $\left\{\psi,[\psi]_{A}^{-} \chi\right\} \subseteq \pi(s)$ imply

$-\chi \in \pi^{\prime}(s)$

- for every $t \in \mathscr{K}_{a}(s)$, for every $b \notin A,\left\{K_{b} \theta \mid K_{b} \theta \in \pi^{\prime}(t)\right\}=\left\{K_{b} \theta \mid K_{b} \theta \in \pi(t)\right\}$, while for every $a \in A,\left\{K_{a} \theta \mid K_{a} \theta \in \pi^{\prime}(t)\right\}=\left\{K_{a} \theta \mid K_{a} \theta \in \pi(t)\right\} \cup\left\{K_{a} \theta \mid K_{a} \theta \in \operatorname{Sub}_{C}^{+}\left(K_{a} \psi\right)\right\}$ iff $\psi \in \pi(t)$ and $\left\{K_{a} \theta \mid K_{a} \theta \in \pi^{\prime}(t)\right\}=\left\{K_{a} \theta \mid K_{a} \theta \in \pi(t)\right\} \cup\left\{K_{a} \theta \mid K_{a} \theta \in \operatorname{Sub}_{C}^{+}\left(K_{a} \neg \psi\right)\right\}$ iff $\neg \psi \in \pi(t)$;

- for every $t \notin \mathscr{K}_{a}(s), \pi^{\prime}(t)=\pi(t)$.

- each $R_{(s, \psi, A)}^{+}$is a relation on epistemic structures such that $R_{(s, \psi, A)}^{+}\left(M, M^{\prime}\right)$ and $\left\{\psi,[\psi]_{A}^{+} \chi\right\} \subseteq \pi(s)$ imply

$-\chi \in \pi^{\prime}(s)$

- for every $t \in\left(\bigcup_{a \in A} \mathscr{K}_{a}\right)^{*}(s)$, for every $b \notin A,\left\{K_{b} \theta \mid K_{b} \theta \in \pi^{\prime}(t)\right\}=\left\{K_{b} \theta \mid K_{b} \theta \in \pi(t)\right\}$, while for every $a \in A,\left\{K_{a} \theta \mid K_{a} \theta \in \pi^{\prime}(t)\right\}=\left\{K_{a} \theta \mid K_{a} \theta \in \pi(t)\right\} \cup\left\{K_{a} \theta \mid K_{a} \theta \in \operatorname{Sub}_{C}^{+}\left(C_{A} \psi\right)\right\}$ iff $\psi \in \pi(t)$ and $\left\{K_{a} \theta \mid K_{a} \theta \in \pi^{\prime}(t)\right\}=\left\{K_{a} \theta \mid K_{a} \theta \in \pi(t)\right\} \cup\left\{K_{a} \theta \mid K_{a} \theta \in \operatorname{Sub}_{C}^{+}\left(C_{A} \neg \psi\right)\right\}$ iff $\neg \psi \in \pi(t)$;

- for every $t \notin\left(\bigcup_{a \in A} \mathscr{K}_{a}\right)^{*}(s), \pi^{\prime}(t)=\pi(t)$.

Clearly, if any tableau $T$ for a formula $\phi$ as above is non-empty, then $\phi$ is satisfiable. In particular, we can prove that for every $\psi \in \operatorname{Sub}_{C}^{+}(\phi),(M, s) \models \psi$ iff $\psi \in \pi(s)$, for $s \in M$.

Furthermore, the soundness of the procedure follows by the soundness of the three steps separately. Firstly, $\phi$ is satisfiable as an epistemic formula iff then there exists at least one epistemic model $M$ defined as above. Given $M$, there are finitely many $M^{\prime}$ non \pm -bisimilar to $M$. Then, we attempt to build a GLAL tableau $T$ starting in each of these $M^{\prime}$. In particular, $T$ is not empty iff $\phi$ is satisfiable.

\footnotetext{
${ }^{1}$ In [17] $\operatorname{Sub}(\phi)$ is the set of all subsets of $\phi ; \operatorname{Sub}_{C}(\phi)$ extends $\operatorname{Sub}(\phi)$ with formulas $E(\psi \wedge C \psi)$ and $\psi \wedge C \psi$ for every subformula $\psi \in \operatorname{Sub}(\phi)$; and $\operatorname{Su} b_{C}^{+}(\phi)$ includes $\operatorname{Sub} b_{C}(\phi)$ and the negation of formulas in $\operatorname{Sub}_{C}(\phi)$.
} 